\title{
The 5th International p63/p73 workshop: much more than just tumour suppression
}

\author{
MP Kadakia ${ }^{*, 1}$, CC de-Fromentel ${ }^{*, 2,3,4}$ and K Sabapathy ${ }^{*, 5}$ \\ Cell Death and Differentiation (2012) 19, 549-550; doi:10.1038/cdd.2011.204; published online 13 January 2012
}

The 5th p63/p73 workshop, the International Agency for Research on Cancer, Lyon, France, September 12-14, 2011

The 5th p63/p73 workshop was held in Lyon, France, at the International Agency for Research on Cancer, from September 12-14, 2011, with 62 oral and 24 poster presentations. This year's workshop highlighted the multitude of actions exerted by $p 63$ and p73, especially non-traditional isoformspecific roles, as well as the description of several mutant mouse models and genome-wide association studies, improving our understanding of diverse topics like development, metabolism, cancer initiation and invasion. The workshop clearly demonstrated that research on the different p53 family members is more intertwined and complex than ever thought before. Much like the city of Lyon, a mixture of modern technology and historical wonder, the p63/p73 fields continue to blend together our understanding of how development and disease complement one another.

\section{Stem cells (SCs) and Development}

Involvement of the p53 family polymorphisms in regulating the genomic stability of germ-lines, and developmental defects in eggs and autism in offspring, demonstrated a key role for these proteins in governing germ-line stability and embryonic development, beyond cancer. Specialized roles for these family members in regulating tissue-specific development and differentiation were an emerging theme. For example, TAp73 was reported to be involved in maintaining the embryonic and adult SC numbers by inhibiting neurogenesis via induction of Hey2 expression, in addition to activation of miR34a, which led to decreased synaptogenesis. On the other hand, $\Delta \mathrm{Np} 73$ is involved in neuronal viability, by inhibiting the p53-mediated inhibition of self-renewal. $\Delta \mathrm{Np63}$ was also reported to be important for the survival of neural SCs, as its knock down was shown to result in SC apoptosis.

TAp63-null ES cells were unable to generate cardiomyocytes, which was consistent with regulation of $S O X-17, A C T-A$ and GATA4/6 by TAp63 in endodermal cells involved in cardiogenesis. p38MAPK/p53 pathway was shown to be involved in the commitment to mesodermal lineages, especially, to the cardiac fate via regulation of the intracellular levels of $\mathrm{p} 53$.

$\triangle \mathrm{Np63}$ was demonstrated to have an important role in miRNA processing by activating DGCR8 expression, which processes miRNAs targeting OCT4, SOX2 and NANOG genes required for SC pluripotency - thereby favouring commitment to epidermal differentiation.

\section{Targets and Regulators}

Many other presentations focused on dissecting the targets and regulators of $p 63 / p 73$. In keratinocytes, $\Delta N p 63$ increases RUNX1 expression during proliferation, whereas p53 downregulates RUNX1 during differentiation via an independent response element. Contrary to RUNX1, $\Delta N p 63 \alpha$ negatively regulates PTEN expression, and expression levels of both $\triangle \mathrm{Np63} \alpha$ and PTEN were found to be deregulated in non-melanoma skin cancers. p63 influences keratinocyte differentiation through induction of mir-17 family members, while p63 itself was downregulated by microRNAs induced by iASPP. Through the interaction and forced translocation of $\mathrm{YB}-1$ into the nucleus, $\Delta \mathrm{Np} 63 \alpha$ reduced the stabilization of SNAIL and cell motility. Moreover, $\Delta \mathrm{Np} 63 \alpha$ was reported to reduce cell migration in breast cancer cell lines through MAP Kinase Phosphatase 3 (MPK3)-mediated repression of ERK1/2 activation. However, MCF10A cells cultured in conditioned media from mammary tumor stroma exhibited increased proliferative potential because of enhanced $\Delta \mathrm{Np} 63 \alpha$ expression, highlighting the context-specific functions of p63. Analysis of $\mathrm{TAp} 3^{-1-}$ mice revealed that they are obese and diabetic through improper regulation of SIRT1 and AMPK $\alpha$.

Focusing on p73, it was shown that HPV38 is capable of inducing transformation through both p53-mediated induction and IKK $\beta$-mediated stabilization of $\Delta \mathrm{Np} 73$. TAp $73^{-/-}$mice

\footnotetext{
${ }^{1}$ Department of Biochemistry and Molecular Biology, Wright State University, Dayton, OH, USA; ${ }^{2}$ INSERM U1052, Centre de Recherche en Cancérologie de Lyon, Lyon, France; ${ }^{3}$ CNRS5286, Centre de Recherche en Cancérologie de Lyon, Lyon, France; ${ }^{4}$ Université Lyon-1, Lyon, France and ${ }^{5}$ Division of Cellular \& Molecular Research, Humphrey Oei Institute of Cancer Research, National Cancer Centre, Singapore, Singapore

${ }^{*}$ Corresponding authors: MP Kadakia, Department of Biochemistry and Molecular Biology, Wright State University, Colonel Glenn Highway, Dayton, OH 45435, USA. Tel: + 1937775 2339; Fax: + 1937775 3730; E-mail: madhavi.kadakia@ wright.edu; CC de-Fromentel, Centre de Recherche en Cancérologie de Lyon, UMR INSERM U1052 CNRS5286, 151, cours Albert Thomas, 69003 Lyon, France. Tel: + 3347268 1957; Fax: + 3347268 1971; E-mail: claude.de-fromentel@ inserm.fr and K Sabapathy, Division of Cellular \& Molecular Research, Humphrey Oei Institute of Cancer Research, National Cancer Centre, 11, Hospital Drive, Singapore 169610, Singapore. Tel: + 656436 8349; Fax: + 656226 5694; E-mail: cmrksb@nccs.com.sg
} 
when crossed into a model of pancreatic ductal adenocarcinoma succumb to accelerated tumorigenesis, due to high levels of aneuploidy and invasion. Furthermore, the ability of DNA damage to induce the activation of $p 73$ is dependent on MST1/LATS1/2-mediated phosphorylation and cell density dependent cytoplasmic sequestration of the YAP adaptor protein.

\section{Tumor, Ageing and Senescence}

A substantial amount of data was presented to highlight the critical roles for $p 63$ and $p 73$ in regulating senescence. Both $\mathrm{TAp} 3^{-1-}$ and TAp63 ${ }^{+/-}$mice exhibited increased senescence and reduced lifespan. Absence of either TAp73 or TAp63 led to accelerated senescence in MEFs, with several microRNA targets being identified as p63 targets. Consistently, ras-induced senescence was abrogated in TAp63 ${ }^{-1-}$ MEFs, or upon overexpression, $\Delta \mathrm{Np} 63$ promoted growth by regulating c-rel. TAp73 ${ }^{-1-}$ mice were reported to have reduced subcutaneous fat and cellular metabolic profile was markedly affected by TAp73 expression.

Although TAp63 regulates oocyte survival via induction of PUMA, a new retrovirally driven p63 (known as GTAp63) was identified to be expressed mainly in the male germline, confers sensitivity upon cisplatin treatment. Moreover, inhibition of p63's function of dicer transactivation and subsequent regulation of $\mathrm{MET}$ signalling by mutant p53 resulted in negating its inhibitory functions on migration. Absence of TAp63 did not have a profound effect on tumorigenesis even in the TPA/DMBA model, suggesting that TAp63 loss was not sufficient to promote tumourigenesis. Nonetheless, TAp63 absence was sufficient to promote metastasis owing to dicer deficiency. Similar growth regulatory properties of TAp73 were also highlighted in the context of $H$. pylori infection, which resulted in an increase of TAp73 invoking an apoptotic response in host cells. These data together demonstrate the tumour suppressive role for TAp73 and TAp63 proteins in different contexts. Conversely, overexpression of $\Delta \mathrm{Np} 63$ with ras accelerated carcinoma formation, leading to identification of new $\Delta$ Np63-specific target genes involved in pro-survival and anti-senescence. Absence of $\Delta \mathrm{Np} 73$ or $\Delta \mathrm{Np} 63$ consistently led to suppression of thymic lymphomagenesis or murine head and neck squamous cell carcinoma (HNSCC) because of p53 loss. $\Delta$ Np63, via regulation of the FGFR members, promotes migration and cellular invasion, further supporting the tumour-promoting role of the $\Delta \mathrm{N}$ proteins.

On the flip side, TAp73 was shown to promote angiogenesis upon hypoxia, and both TAp73 and TAp63 were also shown to transactivate the short-anti-apoptotic p53 isoform, $\Delta 133$. In addition, beta-catenin activates $\Delta \mathrm{Np} 63$, highlighting a potential mechanism contributing to tumourigenesis. p63 expression was shown to have a poor prognosis in melanoma patients, and silencing p63 expression promoted sensitivity to cisplatin treatment. Similarly, $\Delta$ Np63 was reported to affect TAp73 function in HNSCCs. These data together also suggest that the TAp73 and TAp63 proteins may have crucial roles in supporting cellular survival.

\section{Pathology}

Several studies were presented to understand the differential binding of mutant p63 when compared with wild-type p63 using ChIP-Seq and microarray. Using TP63 ${ }^{-1-}$ mice more than 250 genes mis-regulated in the $\mathrm{TP} 63^{-/-}$palates were identified, several of them involved in tight junction formation, cellular adhesion and Wnt signaling pathway. More than 8000 high stringency p63-binding sites were found, many of them in regulatory elements far removed $(>10 \mathrm{~kb})$ from the respective transcription start site of genes. A subset of split hand/foot formation patients had no p63 mutations but who instead had deletions of the p63-binding site over $250 \mathrm{~kb}$ away from the $D L X 6 / 5$ gene, a p63 target gene. Some of the genes containing p63-binding sites are genetically linked to cleft palate. Interestingly, AP- $2 \alpha$, the mutation of which is associated with craniofacial abnormalities and cleft palate, was identified as a co-regulator of many of these genes. Genomewide analysis demonstrated an overlap in p63-binding sites with histone modifications indicative of active enhancer elements during the proliferative stages of keratinocyte culture that declined during differentiation. Similarly, p63-binding sequences also overlapped mutant p53-binding sites, thereby suggesting co-regulation of common targets.

Besides, studies on the generation of several mutant p63 mouse models were presented. Heterozygous mutation of p63L514F in mice recapitulated human AnkyloblepharonEctodermal defects-Cleft lip/palate syndrome with 100\% penetrance, in part due to reduced proliferation from deregulated FGFR2 signalling. Generation of an Ectrodactyly-Ectodermal dysplasia-Cleft syndrome mouse model i.e., p63R $279 \mathrm{H}$ knock-in - demonstrated that perinatal lethality, craniofacial defects and skin morphology phenotypes were dependent on the mouse strain and the presence of the neomycin cassette within intron 4 (hence, hypomorphic allele). The K5-p63R298Q mutant mice - a model for AcroDermato-Ungual-Lacrimal-Tooth syndrome showed a simi-

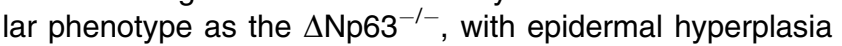
and hair abnormalities, amongst other defects.

A critical insight into the different structures of the proapoptotic members of the p63/p73 family was also presented, demonstrating the vast differences between members of the p53 family. Unlike other p63 isoforms, TAp63 $\alpha$ exists as an inactive dimer, which upon phosphorylation leads to uncoupling of the transcription inhibitory domain from the transactivation domain to form an irreversible, transcriptionally active tetramer, which then promotes oocyte apoptosis.

\section{Conflict of Interest}

The authors declare no conflict of interest.

Acknowledgements. We would like to thank Cell Death and Differentiation Conferences, National Cancer Centre Singapore, Cell Death and Disease, Synergie Lyon Cancer Foundation, International Agency for Research on Cancer and Centre de Recherche en Cancérologie de Lyon for their support, and the organizers and chairs for putting together a productive, engaging workshop. 